\title{
An Integrated 5 GHz Wideband Quadrature Modem for OFDM Gbit/s Transmission in SiGe:C BiCMOS
}

\author{
Klaus Schmalz, Eckard Grass, Frank Herzel, and Maxim Piz \\ Received 25 January 2007; Revised 23 April 2007; Accepted 3 August 2007 \\ Recommended by Kenjiro Nishikawa \\ This paper presents a $5 \mathrm{GHz}$ wideband I/Q modulator/demodulator for $650 \mathrm{MHz}$ OFDM signal bandwidth, which is integrated \\ with a $5 \mathrm{GHz}$ phase locked loop for I/Q generation. The quadrature signals are derived from a $10 \mathrm{GHz}$ CMOS VCO followed by \\ a bipolar frequency divider. The phase noise at $1 \mathrm{MHz}$ offset is $-112 \mathrm{dBc} / \mathrm{Hz}$ for the modulator as well as for the demodulator. \\ The chips were produced in a $0.25 \mu \mathrm{m} \mathrm{SiGe} \mathrm{BiCMOS} \mathrm{technology.} \mathrm{The} \mathrm{signal-to-noise} \mathrm{ratio} \mathrm{(SNR)} \mathrm{of} \mathrm{transmitted/received} \mathrm{OFDM}$ \\ signal and the corresponding I/Q mismatch versus baseband frequency are given. The modulator achieves an SNR of 22-23 dB, \\ and the demodulator realizes an SNR up to $22 \mathrm{~dB}$. The modulator reaches a data rate of $2.16 \mathrm{Gbit} / \mathrm{s}$ using 64 QAM OFDM, and the \\ demodulator realizes $1.92 \mathrm{Gbits} / \mathrm{s}$.
}

Copyright () 2007 Klaus Schmalz et al. This is an open access article distributed under the Creative Commons Attribution License, which permits unrestricted use, distribution, and reproduction in any medium, provided the original work is properly cited.

\section{INTRODUCTION}

High-performance wireless communication systems based on OFDM require significant implementation effort for the RF front-end. In particular, low-phase noise, high linearity, and accurate quadrature matching are needed. For a direct conversion transceiver, one of the main challenges is to realize accurate I/Q phase and amplitude balance. In case of wideband applications, these challenges are even harder, since the generation of an accurate quadrature local oscillator signal is more difficult. This also applies to modulators and demodulators in the $5 \mathrm{GHz}$ band, and to our $60 \mathrm{GHz}$ transceiver where $5 \mathrm{GHz}$ is used as an intermediate frequency [1].

For RF circuit design, SiGe BiCMOS technology [2] is beneficial for several reasons. First, the CMOS compatibility allows high integration resulting in lower cost compared to compound semiconductor technologies. Second, the noise behavior and power consumption compare favorably with CMOS technologies. Recently, a fully integrated $5 \mathrm{GHz}$ quadrature demodulator [3] and modulator [4] in SiGe technologies have been presented. In these papers, polyphase filters have been used for I/Q generation. In [4], a $5 \mathrm{GHz}$ SiGe quadrature modulator, which contains a circular polyphase filter, for $1 \mathrm{Gbit} / \mathrm{s}$ transmission with 64 QAM OFDM with a signal bandwidth of $204 \mathrm{MHz}$ has been published. Because for a single polyphase filter, an accurate I/Q generation is limited to a relatively narrow frequency range, multistage polyphase filter design is used for wideband ap- plications. A $0.75-3.6 \mathrm{GHz}$ SiGe quadrature modulator with a 5-stage polyphase filter has been reported [5].

To avoid the difficult and area-consuming polyphase filter design, frequency division by two can also be used for I/Q generation [6-9]. This technique is well suited for wideband and multiband applications, as the accurate I/Q generation is not limited to a relatively narrow frequency band. Moreover, since this approach utilizes a VCO running at twice the local oscillator (LO) frequency, the LO pulling effect in the transceiver is avoided. However, the divide-by-two technique needs a differential VCO signal without significant amplitude and phase errors, respectively, which can only be delivered by an on-chip integrated VCO.

This paper presents an integrated wideband quadrature modem (modulator and demodulator) for wideband OFDM, where, in contrast to [4], the PLL for I/Q generation is fully integrated with the quadrature modulator/demodulator; and frequency division is used to apply an OFDM signal bandwidth of $650 \mathrm{MHz}$. The mean signal-tonoise ratios (SNR) on the subcarrier level and the corresponding I/Q mismatch, characterized by sideband suppression, are used to evaluate the performance of the $5 \mathrm{GHz}$ modulator and the demodulator.

The $5 \mathrm{GHz}$ quadrature modulator achieves error-free data transmission at $2.16 \mathrm{Gbit} / \mathrm{s}$ using $64 \mathrm{QAM}$ OFDM-based transmission scheme similar to the 802.11a standard, but with $650 \mathrm{MHz}$ signal bandwidth. The $5 \mathrm{GHz}$ demodulator allows error-free transmission at a rate of $1.92 \mathrm{Gbit} / \mathrm{s}$ using 64 QAM OFDM. 


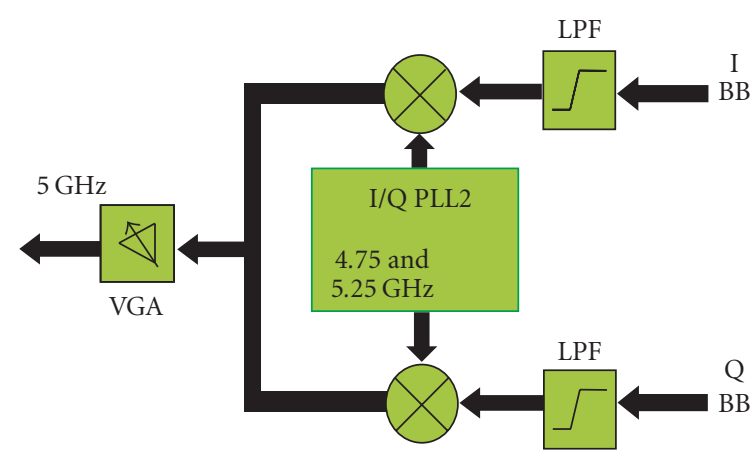

Figure 1: Schematic view of the $5 \mathrm{GHz}$ modulator.

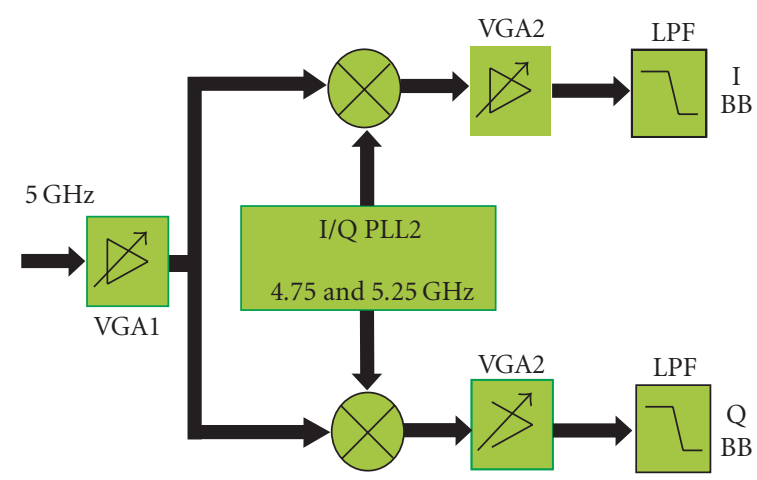

FIGURE 2: Schematic view of the $5 \mathrm{GHz}$ demodulator.

\section{CIRCUIT TOPOLOGY}

In our $60 \mathrm{GHz}$ OFDM demonstrator, the signal is converted from $5 \mathrm{GHz}$ to $61 \mathrm{GHz}$ and vice versa using a $56 \mathrm{GHz}$ PLL, as described in [1]. As shown in Figure 1, the conversion from baseband to $5 \mathrm{GHz}$ requires a wideband quadrature PLL, a single-sideband mixer, a variable-gain amplifier, and integrated lowpass filters of tunable cutoff frequency.

The $5 \mathrm{GHz}$ demodulator topology requires similar components as the $5 \mathrm{GHz}$ modulator [8], as shown in Figure 2 . In order to process two $500 \mathrm{MHz}$ bands from $60.5 \mathrm{GHz}$ to $61.5 \mathrm{GHz}$, the IF PLL must generate $4.75 \mathrm{GHz}$ and $5.25 \mathrm{GHz}$ [1].

The inputs and outputs of the converters are differential. The wideband PLL is to deliver I/Q signals at 5.25 and $4.75 \mathrm{GHz}$. This results in a required tuning range of $500 \mathrm{MHz}$ plus some margin for compensation of temperature and process variations.

\section{DESIGN OF SUBCIRCUITS}

For I/Q generation, a bipolar divide-by-two circuit (DTC) is used [9]. By this mean, the good phase noise performance of a MOS VCO due to the large internal signal swing is combined with the low-power consumption of SiGe dividers. Figure 3 shows the DTC realized as two latches in a negative feedback loop, and the latch, which is composed of a differential pair and a regenerative pair. The DTC draws a current of $2.7 \mathrm{~mA}$ from a $2.5 \mathrm{~V}$ supply.
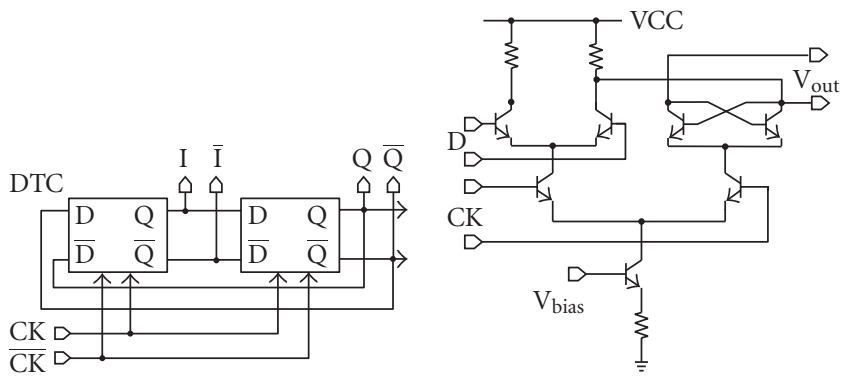

FIGURE 3: Schematics of divide-by-two circuit and the latch.

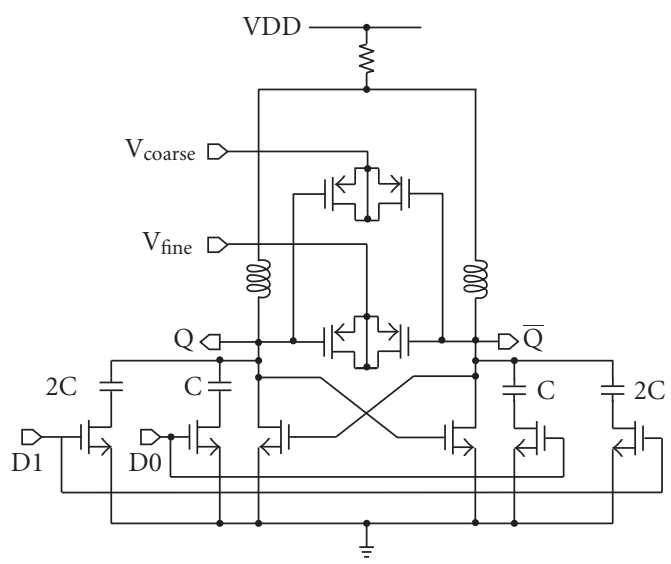

FIGURE 4: Schematic view of $10 \mathrm{GHz}$ MOS VCO.

Figure 4 presents the schematic view of the $10 \mathrm{GHz}$ VCO. The MOS oscillator has two digital control inputs for subband selection, which reduces the VCO gain for low-phase noise.

Further reduction of phase noise and spurs maintaining a large tuning range is achieved by using coarse and fine tuning in conjunction with a dual-loop PLL shown in Figure 5 as described in [10]. The PLL has a $5 \mathrm{MHz}$ reference input. The dashed box symbolizes the $10 \mathrm{GHz}$ VCO with coarse and fine tuning input followed by the DTC.

The quadrature mixers in the modulator and demodulator consist of two linearized Gilbert mixers including a buffer amplifier as described in [11]. Figure 6 depicts the mixer and the buffer of the $5 \mathrm{GHz}$ modulator. In contrast to [11], the mixer does not use additional transistors for linearization. The $4.75 \mathrm{GHz} / 5.25 \mathrm{GHz}$ signals from the PLL are ac coupled into the LO inputs of the two mixers.

Figure 7 presents the $5 \mathrm{GHz}$ variable gain amplifier (VGA) cell, which consists of a transadmittance stage followed by a transimpedance stage with internal Ac coupling to enable supply voltage of $3.3 \mathrm{~V}$. The first stage includes a variable-gain bipolar quad, which is similar to a Gilbert mixer. The gain is controlled by the voltage difference VC, which is converted from an external control bias VG using an additional internal converter. The output signal of the VGA is connected to an output buffer, which is formed as differential pair with resistive emitter degeneration. 


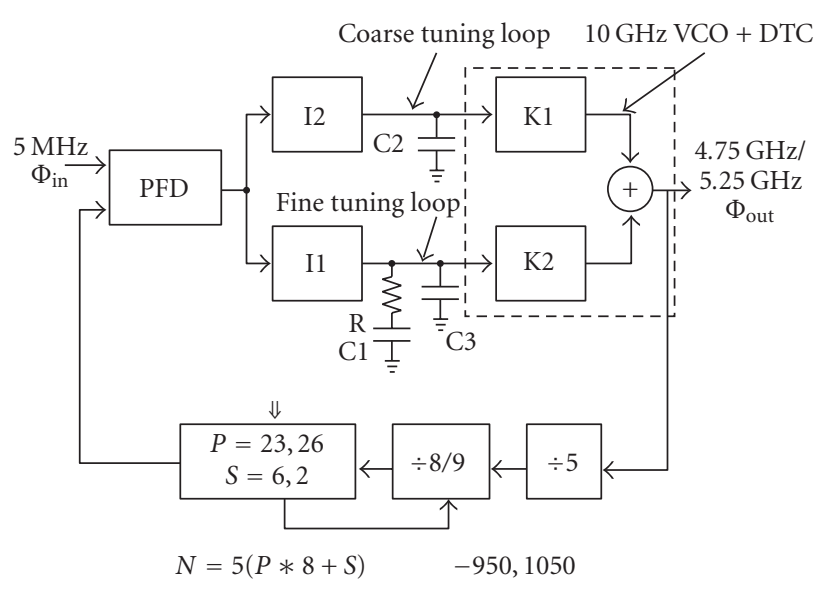

FIgURe 5: Dual-loop PLL.

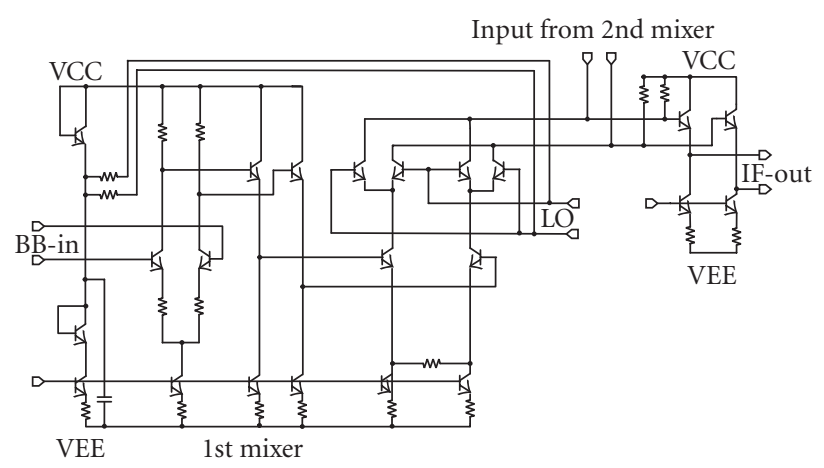

FIgURE 6: Schematics of mixer and buffer.

In case of the demodulator, the $5 \mathrm{GHz}$ VGA is composed of two cascaded VGA cells with internal Ac coupling. The second VGA (VGA2) of the $5 \mathrm{GHz}$ demodulator is built as variable-gain bipolar quad, buffered by emitter followers.

For the lowpass filter (LPF), a differential log-domain (LD) LPF with a tunable cutoff frequency of $100-500 \mathrm{MHz}$ was applied using a sixth-order Butterworth filter with cascaded biquads [12]. Figure 8 illustrates the block diagram of the LD filter with the three cascaded biquads (bq1, bq2, and bq3), the rectifier for class $A B$ operation $(A B)$, the current source bank (CS) for biasing the biquads, the single-ended voltage-to-current converters $(\mathrm{V} / \mathrm{I})$ at the input, and the differential current-to-voltage converter (I/V) at the output.

\section{EXPERIMENTAL RESULTS}

Figure 9 shows the chip micrograph of the $5 \mathrm{GHz}$ modulator, and Figure 10 shows the chip micrograph of the $5 \mathrm{GHz}$ demodulator. The chips were fabricated in a $0.25 \mu \mathrm{m}$ highperformance SiGe:C technology with $\mathrm{f}_{\mathrm{t}} / \mathrm{f}_{\max }=200 / 200 \mathrm{GHz}$ [13].

The area of the modulator chip is $1.3 \times 3.2 \mathrm{~mm}^{2}$, and the area of the demodulator chip is also $1.3 \times 3.2 \mathrm{~mm}^{2}$. A significant amount of the chip area is due to the PLL and the integrated lowpass filters. Optionally, for chip testing, these lowpass filters can be bypassed. The I/Q PLL consumes $57 \mathrm{~mA}$ at

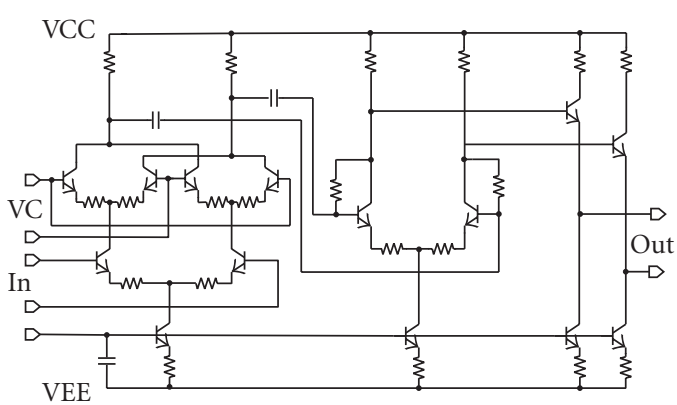

FIGURE 7: Schematic view of $5 \mathrm{GHz}$ variable gain amplifier.

$2.5 \mathrm{~V}$ supply voltage, and the quadrature mixer draws $13 \mathrm{~mA}$ at $3.0 \mathrm{~V}$. The single-sideband output spectrum of the modulator is presented in Figure 11, which was measured with Agilent E4440A spectrum analyzer. The spur levels for the modulator as well as for the demodulator are as low as -73 $\mathrm{dBc}$, despite the large PLL tuning range of $1 \mathrm{GHz}$.

Figure 12 shows the modulator output spectrum for a sinusoidal I/Q input of $120 \mathrm{MHz}$. The I/Q baseband signal was generated by Agilent N6030A arbitrary waveform generator.

In this case, a single sideband (SSB) modulation is performed as the $5 \mathrm{GHz}$ modulator corresponds to an SSB mixer, where the LO signal in quadrature is delivered by the quadrature PLL. The main signal is at $5.13 \mathrm{GHz}$ for the LO signal at $5.25 \mathrm{GHz}$. The sideband is located at $5.37 \mathrm{GHz}$. The sideband suppression is $-36 \mathrm{dBc}$. Figure 13 shows the complex baseband signal at the output of the demodulator in case of a $5.25 \mathrm{GHz}$ input signal, which is SSB-modulated by an I/Q $10 \mathrm{MHz}$ sinusoidal signal. The input signal was generated by Agilent E8267D vector signal generator modulated by Agilent N6030A arbitrary waveform generator. The SSB modulated input signal is downconverted by the $5 \mathrm{GHz}$ demodulator to the baseband. The baseband signal was measured by Agilent DSO80804B oscilloscope and analyzed by Agilent 89600 vector signal analyzer (VSA) software. The positive signal is at $10 \mathrm{MHz}$, and the negative signal is at $-10 \mathrm{MHz}$. The corresponding sideband suppression is $-33 \mathrm{dBc}$.

Figure 14 presents the uncalibrated sideband suppression for the modulator and demodulator as a function of the baseband (BB) frequency.

For the modulator, the sideband suppression is in the range from about $-40 \mathrm{dBc}$ to $-30 \mathrm{dBc}$, and for the demodulator in the range from $-33 \mathrm{dBc}$ to $-25 \mathrm{dBc}$. Figure 15 shows the output power of the modulator as a function of the amplitude of the I/Q baseband signals. The gain is controlled by the external bias signal VG. The output P1dB is $-7 \mathrm{dBm}$ for the maximum gain of the modulator. The phase noise at $1 \mathrm{MHz}$ offset is $-112 \mathrm{dBc} / \mathrm{Hz}$ for the modulator as well as for the demodulator, as measured by Agilent E4440A spectrum analyzer using the phase noise option.

\section{OFDM DATA TRANSMISSION}

The OFDM-based transmission scheme of the demonstrator is similar to the 802.11a standard. The signal bandwidth is around $650 \mathrm{MHz}$. Convolutional $(171,133)$ codes are used 


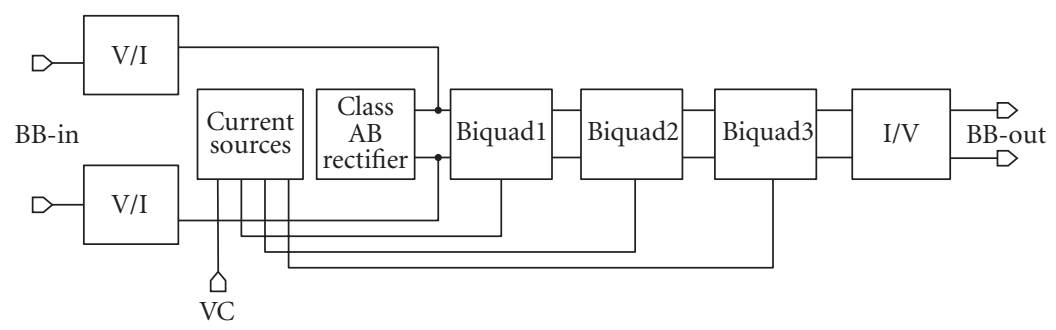

Figure 8: Schematic view of log-domain lowpass filter.

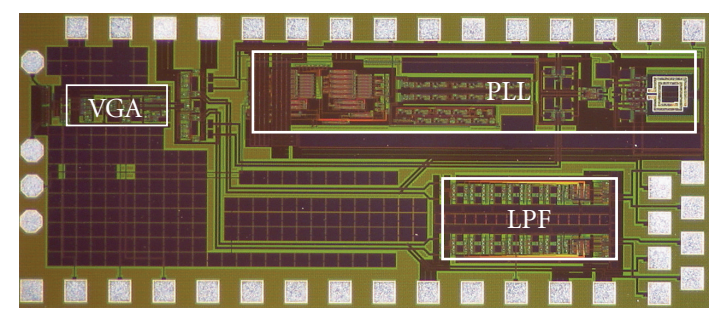

Figure 9: Chip micrograph of the $5 \mathrm{GHz}$ modulator.

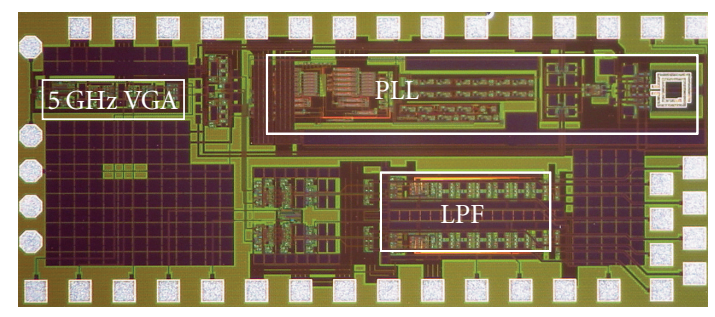

Figure 10: Chip micrograph of the $5 \mathrm{GHz}$ demodulator.

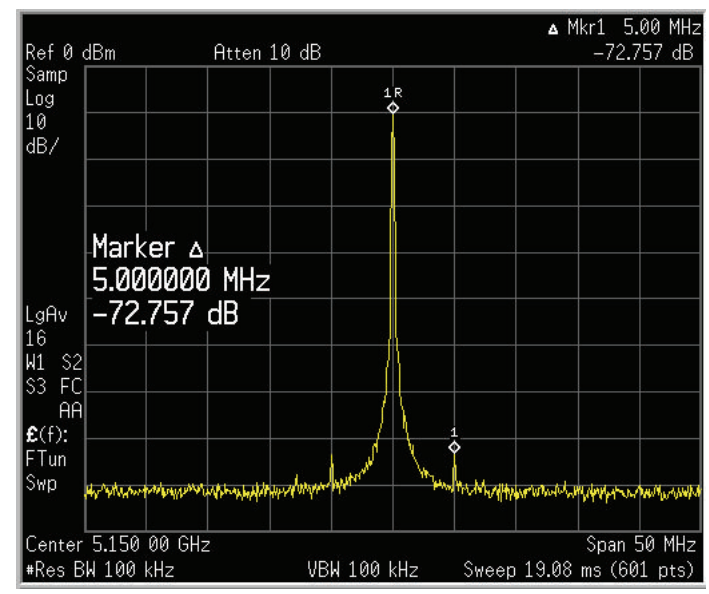

FIGURE 11: Measured modulator output spectrum.

together with fixed transmission modes ranging from BPSK up to 64-QAM. The preamble is extended to eleven OFDM symbols to attain good synchronization and initial channel estimation for low SNR values. The long preamble facilitates accurate channel estimation, and therefore enables very long frames to be transmitted.

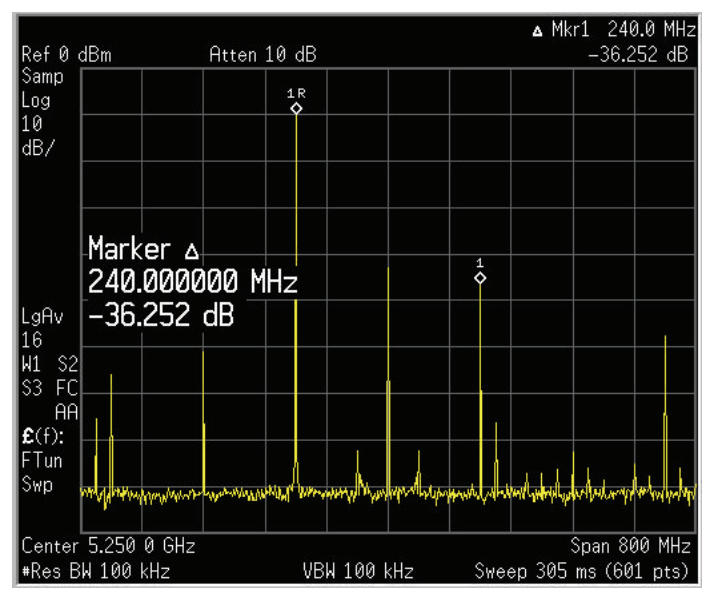

FIGURE 12: Measured modulator output spectrum.

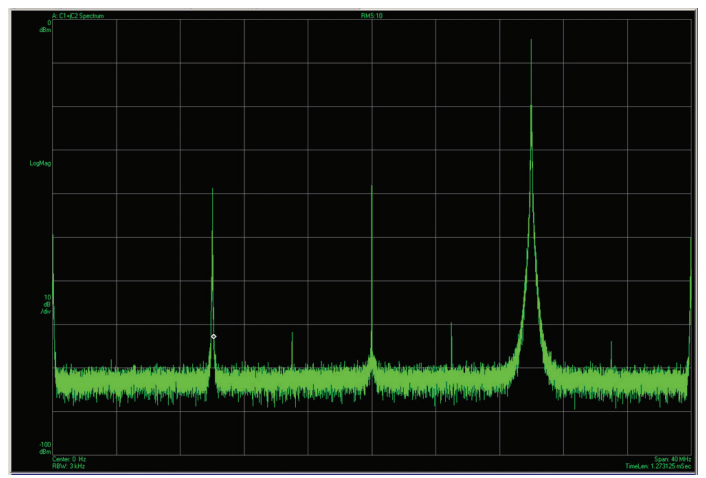

FIGURE 13: Measured demodulator output spectrum (center at $0 \mathrm{~Hz}$, span $40 \mathrm{MHz}$, range from $-100 \mathrm{dBm}$ to $0 \mathrm{dBm}, 10 \mathrm{~dB} / \mathrm{div}$ ).

The basic physical layer OFDM parameters are summarized in Table 1 . The cyclic prefix of $160 \mathrm{~ns}$ was chosen as a good compromise between phase noise sensitivity and maximum tolerable channel delay spread [1]. The raw data rates of the physical layer without taking the preamble into account are summarized in Table 2 for the highest specified data modes.

For testing the data transmission, we used the Agilent N6030A arbitrary waveform generator, the Agilent vector signal generator, and the Agilent $8 \mathrm{GHz}$ oscilloscope with vector signal analyzer (VSA) software to synthesize and record OFDM frames. Each frame has one kilobyte of data. 


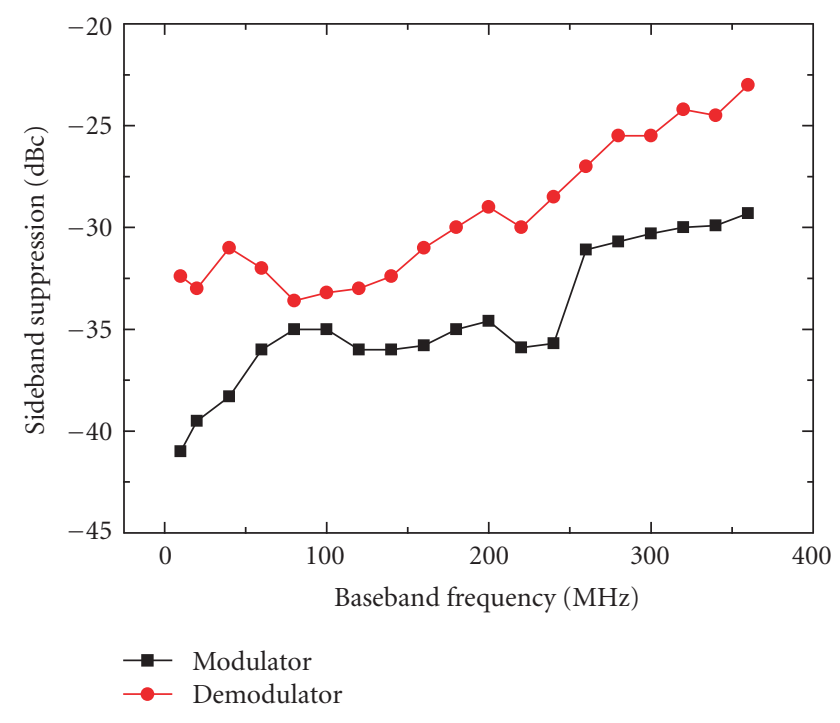

FIGURE 14: Measured uncalibrated sideband suppression.

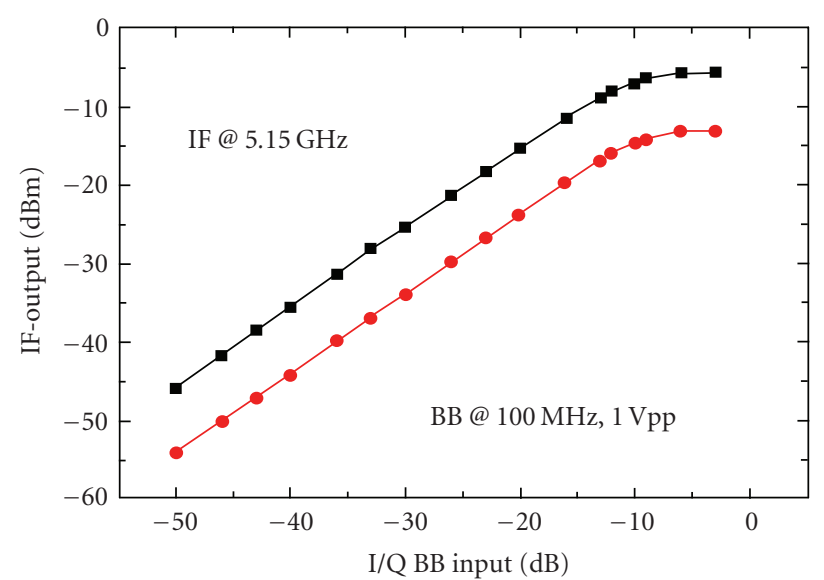

$\rightarrow-\mathrm{VG}=0.5 \mathrm{~V}$

Figure 15: Linearity of $5 \mathrm{GHz}$ modulator.

The generation and evaluation of OFDM signals were performed in software. The modulator and the demodulator were tested alone, and also connected together in a loop. The corresponding setups for these measurements are presented in Figure 16.

The mean signal-to-noise ratio (SNR) on the subcarrier level was taken as a figure of merit.

The $5 \mathrm{GHz}$ modulator achieves an SNR of 22-23 dB independent of the VGA gain, which means of the output power. On the other hand, the $5 \mathrm{GHz}$ demodulator achieves an SNR of up to $22 \mathrm{~dB}$, which depends mainly on the gains of the $5 \mathrm{GHz}$ VGA and the baseband VGA. A lower SNR corresponds to higher gain settings. In the loop configuration, an SNR of $19 \mathrm{~dB}$ was measured for optimized gain and suitable attenuators between the modulator output and the demodulator input. In case of the modulator, we were able to establish error-free transmission using 64-QAM with a code rate
TABLE 1: Basic OFDM parameters.

\begin{tabular}{lccc}
\hline FFT bandwidth & $\begin{array}{c}400 \mathrm{MHz} \\
(800 \mathrm{MHz})\end{array}$ & FFT size & $256(512)$ \\
\hline Signal bandwidth & $\begin{array}{c}330 \mathrm{MHz} \\
(650 \mathrm{MHz})\end{array}$ & Subcarrier spacing & $1.5625 \mathrm{MHz}$ \\
\hline Data subcarriers & $192(384)$ & Symbol duration & $800 \mathrm{~ns}$ \\
\hline Pilot subcarriers & $16(30)$ & Cyclic prefix & $160 \mathrm{~ns}$ \\
\hline DC gap & $7.5 \mathrm{MHz}$ & & \\
\hline
\end{tabular}

TABLE 2: PHY data rates for narrowband and wideband system.

\begin{tabular}{lcc}
\hline Subcarrier constellation & Code rate & Data rate \\
\hline 16-QAM & $1 / 2$ & $480(960) \mathrm{Mbit} / \mathrm{s}$ \\
64-QAM & $2 / 3$ & $960(1920) \mathrm{Mbit} / \mathrm{s}$ \\
64-QAM & $3 / 4$ & $1080(2160) \mathrm{Mbit} / \mathrm{s}$ \\
\hline
\end{tabular}

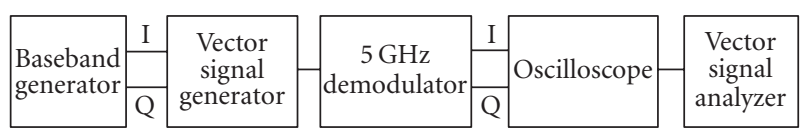

(a)

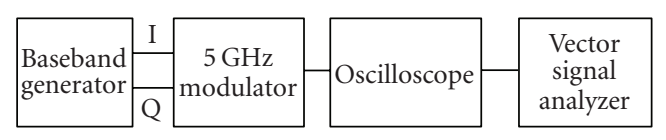

(b)

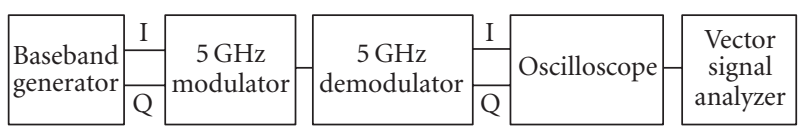

(c)

FIGURE 16: Setups for measurements: (a) demodulator, (b) modulator, and (c) modulator and demodulator in a loop.

of $3 / 4$. This corresponds to a source rate of $2.16 \mathrm{Gbit} / \mathrm{s}$. For the closed loop with $5 \mathrm{GHz}$ modulator and $5 \mathrm{GHz}$ demodulator, we achieved $960 \mathrm{Mbit} / \mathrm{s}$ using 16-QAM-1/2 transmission.

Figure 17 presents the output spectrum of the modulator transmitting the OFDM signal, which was obtained by the VSA software after averaging.

The figures 18, 19, 20 show the signal spectrum, the measured SNR, and the obtained constellation diagram for 64QAM transmission using the $5 \mathrm{GHz}$ modulator only. The figures $21,22,23$ represent the corresponding measurements for the demodulator.

The SNR decreases as function of the subcarrier index for the modulator and demodulator, as shown in Figures 19 and 22. It should be mentioned that this decrease corresponds to 


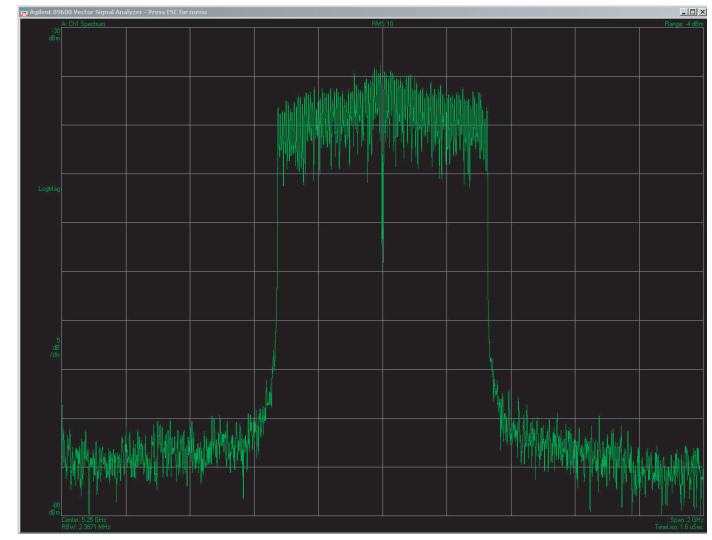

Figure 17: Output spectrum of the modulator (center at $5.25 \mathrm{GHz}$, $2 \mathrm{GHz}$ span, power range from $-80 \mathrm{dBm}$ to $-30 \mathrm{dBm}, 5 \mathrm{~dB} /$ division).

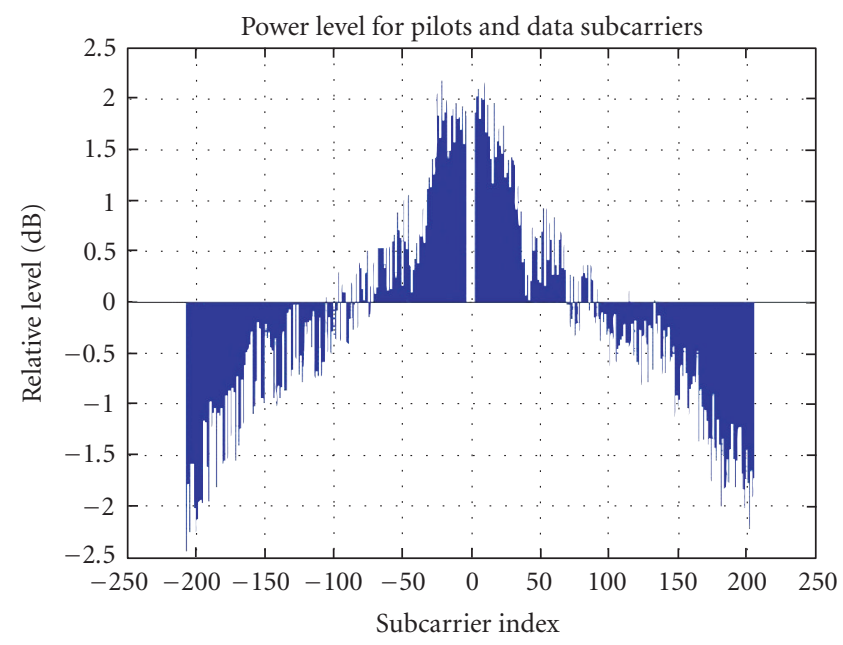

FIGURE 18: Normalized signal spectrum (modulator).

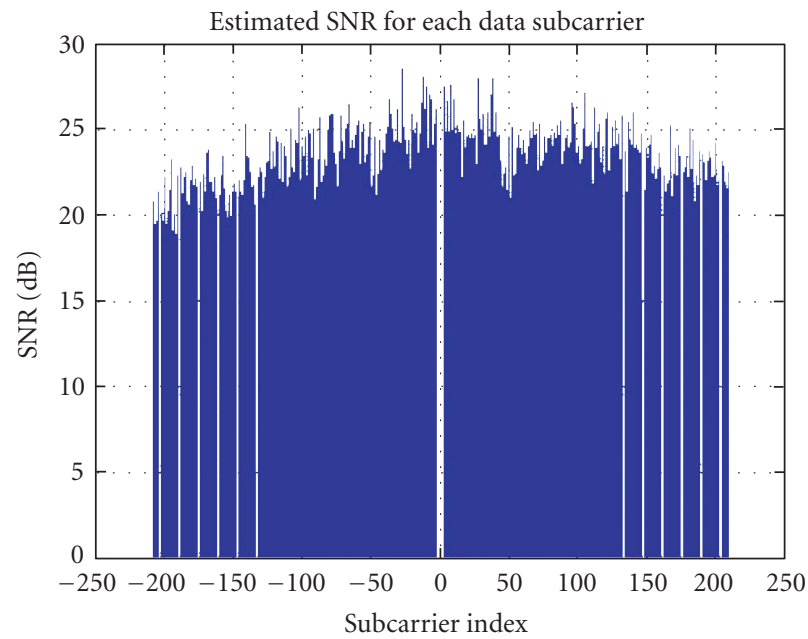

FIGURE 19: Measured SNR for each subcarrier (modulator).

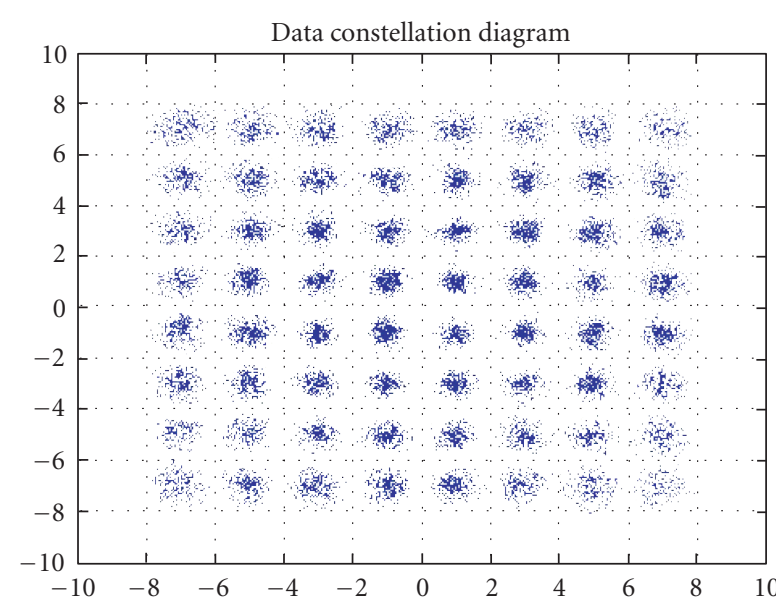

FIGURE 20: 64-QAM constellation diagram (modulator).

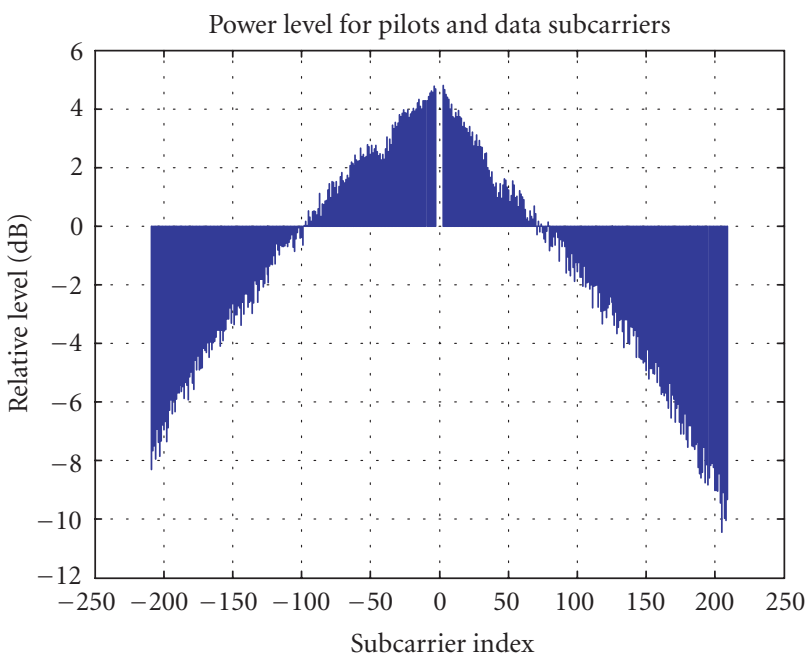

FIGURE 21: Normalized signal spectrum (demodulator).

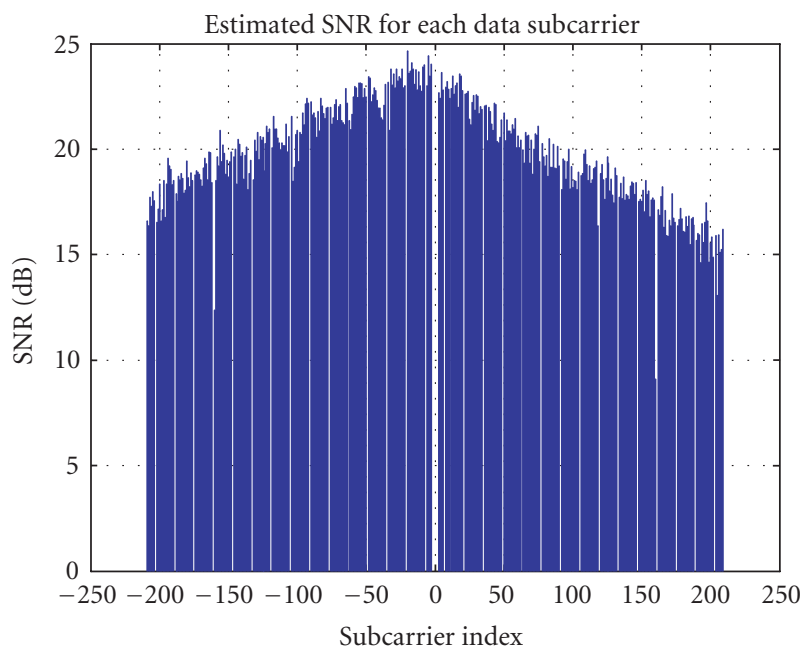

FIGURE 22: Measured subcarrier SNR (demodulator). 


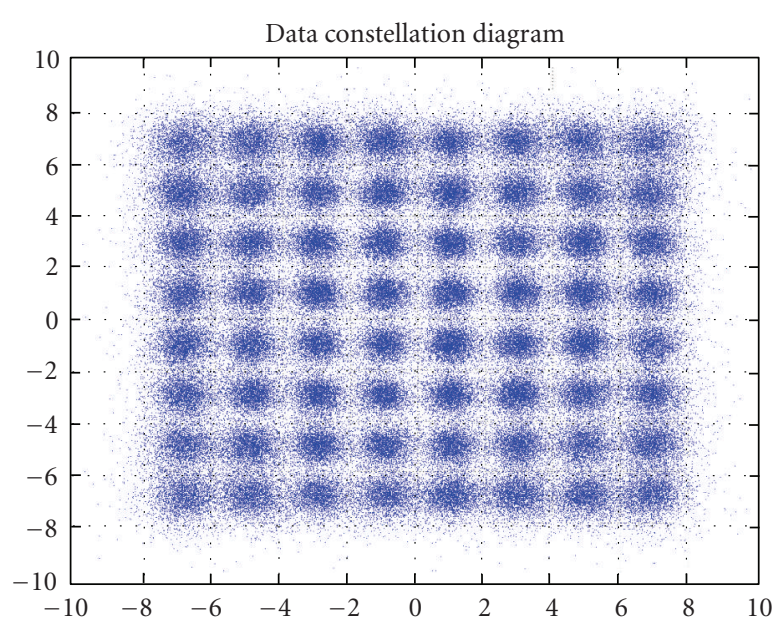

Figure 23: 64-QAM constellation diagram (demodulator).

TABLE 3: Modulator performance for coded and uncoded transmission using wideband OFDM.

\begin{tabular}{lcccc}
\hline Mode & $\begin{array}{c}\text { Coded } \\
\text { BER }\end{array}$ & $\begin{array}{c}\text { Coded } \\
\text { FER }\end{array}$ & $\begin{array}{c}\text { Uncoded } \\
\text { BER }\end{array}$ & Evaluated frames \\
\hline 16-QAM-1/2 & 0 & 0 & $5 \mathrm{e}-4$ & 10 \\
64-QAM-3/4 & 0 & 0 & $2 \mathrm{e}-3$ & 10 \\
\hline
\end{tabular}

TABLE 4: Demodulator performance for coded and uncoded transmission using wideband OFDM.

\begin{tabular}{lcccc}
\hline Mode & $\begin{array}{c}\text { Coded } \\
\text { BER }\end{array}$ & $\begin{array}{c}\text { Coded } \\
\text { FER }\end{array}$ & $\begin{array}{c}\text { Uncoded } \\
\text { BER }\end{array}$ & Evaluated frames \\
\hline 16-QAM-1/2 & 0 & 0 & $1.8 \mathrm{e}-4$ & 23 \\
64-QAM-2/3 & 0 & 0 & $1.3 \mathrm{e}-2$ & 15 \\
64-QAM-3/4 & $3.5 \mathrm{e}-5$ & 0.2 & $1.4 \mathrm{e}-2$ & 32 \\
\hline
\end{tabular}

TABLE 5: Demodulator performance for coded and uncoded transmission using narrowband OFDM.

\begin{tabular}{lcccc}
\hline Mode & $\begin{array}{c}\text { Coded } \\
\text { BER }\end{array}$ & $\begin{array}{c}\text { Coded } \\
\text { FER }\end{array}$ & $\begin{array}{c}\text { Uncoded } \\
\text { BER }\end{array}$ & Evaluated frames \\
\hline 16-QAM-1/2 & 0 & 0 & $\begin{array}{c}\text { BER=3e-6 } \\
\text { FER=0.2 }\end{array}$ & 10 \\
& & & $3 e-3$ & 32 \\
64-QAM-2/3 & 0 & 0 & $3 e-3$ & 18 \\
64-QAM-3/4 & 0 & 0 &
\end{tabular}

the observed lower sideband suppression for larger baseband frequency, see Figure 14.

For the used data modes, the Tables 3,4 , and 5 present the performance data for the modulator and the demodulator, respectively.

For the wideband system, it can be observed that the modulator facilitates error-free transmission at $2.16 \mathrm{Gbit} / \mathrm{s}$ using 64-QAM modulation with a code rate of $3 / 4$, whereas the demodulator allows error-free transmission at a rate of $1.92 \mathrm{Gbit} / \mathrm{s}$ using 64-QAM with a code rate of 2/3.

\section{CONCLUSION}

We have presented an integrated $5 \mathrm{GHz}$ wideband modulator and demodulator in SiGe BiCMOS technology. The generation of the quadrature LO signal is performed by integrating a $10-12 \mathrm{GHz}$ PLL followed by a $1: 2$ frequency divider into the modulator and demodulator. An OFDM data transmission with $2.16 \mathrm{Gbit} / \mathrm{s}$ was achieved for the modulator, $1.92 \mathrm{Gbit} / \mathrm{s}$ was obtained for the demodulator, and $960 \mathrm{Mbit} / \mathrm{s}$ was realized for the modulator connected to the demodulator through suitable attenuators in a loop. The achievable rate for data transmission is limited by the signal-to-noise ratio, which is for the investigated modulator/demodulator mainly due to the I/Q mismatch. The $5 \mathrm{GHz}$ modulator as well as the $5 \mathrm{GHz}$ demodulator can be used in a wideband extension of $5 \mathrm{GHz}$ WLAN systems, but also in a $60 \mathrm{GHz}$ system, where an IF of about $5 \mathrm{GHz}$ is used.

\section{ACKNOWLEDGMENTS}

The authors wish to acknowledge the IHP technology team for chip fabrication. They are indebted to C. Scheytt for support. This work was partly funded by the German Federal Ministry of Education and Research (BMBF) under the project acronym WIGWAM.

\section{REFERENCES}

[1] E. Grass, F. Herzel, M. Piz, Y. Sun, and R. Kraemer, "Implementation aspects of Gbit/s communication system for 60 GHz band," in Proceedings of the 14th Wireless World Research Forum (WWRF '05), San Diego, Calif, USA, July 2005.

[2] B. Heinemann, H. Rücker, R. Barth, et al., "Novel collector design for high-speed SiGe:C HBTs," in Proceedings of IEEE International Electron Devices Meeting (IEDM '02), pp. 775-778, San Francisco, Calif, USA, December 2002.

[3] C. Meng, T.-H. Wu, T.-H. Wu, and G.-W. Huang, "A fully integrated $5.2 \mathrm{GHz}$ double quadrature image rejection Gilbert downconverter using $0.35 \mu \mathrm{m} \mathrm{SiGe} \mathrm{HBT} \mathrm{technology,"} \mathrm{in} \mathrm{Pro-}$ ceedings of the 12th Gallium Arsenide Applications Symposium (GAAS '04), pp. 319-322, Amsterdam, The Netherlands, October 2004.

[4] K. Nakajima, N. Suematsu, K. Murakami, et al., "A 5GHzband SiGe-MMIC quadrature modulator using a circular polyphase filter for 1Gbps transmission," in Proceedings of the 35th European Microwave Conference (EuMC '05), vol. 2, pp. 979-982, Paris, France, October 2005.

[5] E. Tiiliharju and K. Halonen, "A $0.75-3.6 \mathrm{GHz}$ SiGe directconversion quadrature-modulator," in Proceedings of the 29th European Solid-State Circuits Conference (ESSCIRC '03), pp. 565-568, Estoril, Portugal, September 2003.

[6] S. Cipriani, L. Carpineto, B. Bisanti, et al., "Fully integrated zero IF transceiver for GPRS/GSM/DCS/PCS application," in Proceedings of the 28th European Solid-State Circuits Conference (ESSCIRC '02), pp. 439-442, Florence, Italy, September 2002.

[7] F. Herzel, W. Winkler, and J. Borngräber, "An integrated 10 $\mathrm{GHz}$ quadrature LC-VCO in SiGe:C BiCMOS technology for low-jitter applications," in Proceedings of the IEEE Custom Integrated Circuits Conference (CICC '03), pp. 293-296, San Jose, Calif, USA, September 2003. 
[8] K. Schmalz, F. Herzel, and M. Piz, "An integrated $5 \mathrm{GHz}$ wideband quadrature modem in SiGe:C BiCMOS technology," in Proceedings of the 36th European Microwave Conference (EuMC '06), pp. 1565-1659, Manchester, UK, September 2006.

[9] F. Herzel and W. Winkler, "A 2.5-GHz eight-phase VCO in SiGe BiCMOS technology," IEEE Transactions on Circuits and Systems II, vol. 52, no. 3, pp. 140-144, 2005.

[10] F. Herzel, G. Fischer, and H. Gustat, "An integrated CMOS RF synthesizer for 802.11a wireless LAN," IEEE Journal of SolidState Circuits, vol. 38, no. 10, pp. 1767-1770, 2003.

[11] W. Simburger, H. Knapp, and P. Weger, "Characterization of a microwave silicon single-chip direct conversion RF transceiver," in Proceedings of the 25th European Microwave Conference (EuMC '95), vol. 2, pp. 646-657, Bologna, Italy, September 1995.

[12] K. Schmalz, M. A. Teplechuk, and J. I. Sewell, "A class AB 6th order log-domain filter in BiCMOS with 100-500 MHz tuning range," in Proceedings of the European Conference on Circuit Theory and Design (ECCTD '05), vol. 2, pp. 111-114, Cork, Ireland, August-September 2005.

[13] H. Rücker, B. Heinemann, R. Barth, et al., "SiGe:C BiCMOS technology with 3.6 ps gate delay," in IEEE International Electron Devices Meeting Technical Digest (IEDM '03), pp. 5.3.15.3.4, December 2003.

\section{AUTHOR CONTACT INFORMATION}

Klaus Schmalz: IHP-microelectronics, Im Technoloiepark 25,

D-15236 Frankfurt, Germany; schmalz@ihp-microelectronics.com

Eckard Grass: IHP-microelectronics, Im Technoloiepark 25,

D-15236 Frankfurt, Germany; grass@ihp-microelectronics.com

Frank Herzel: IHP-microelectronics, Im Technoloiepark 25,

D-15236 Frankfurt, Germany; herzel@ihp-microelectronics.com

Maxim Piz: IHP-microelectronics, Im Technoloiepark 25,

D-15236 Frankfurt, Germany; piz@ihp-microelectronics.com 

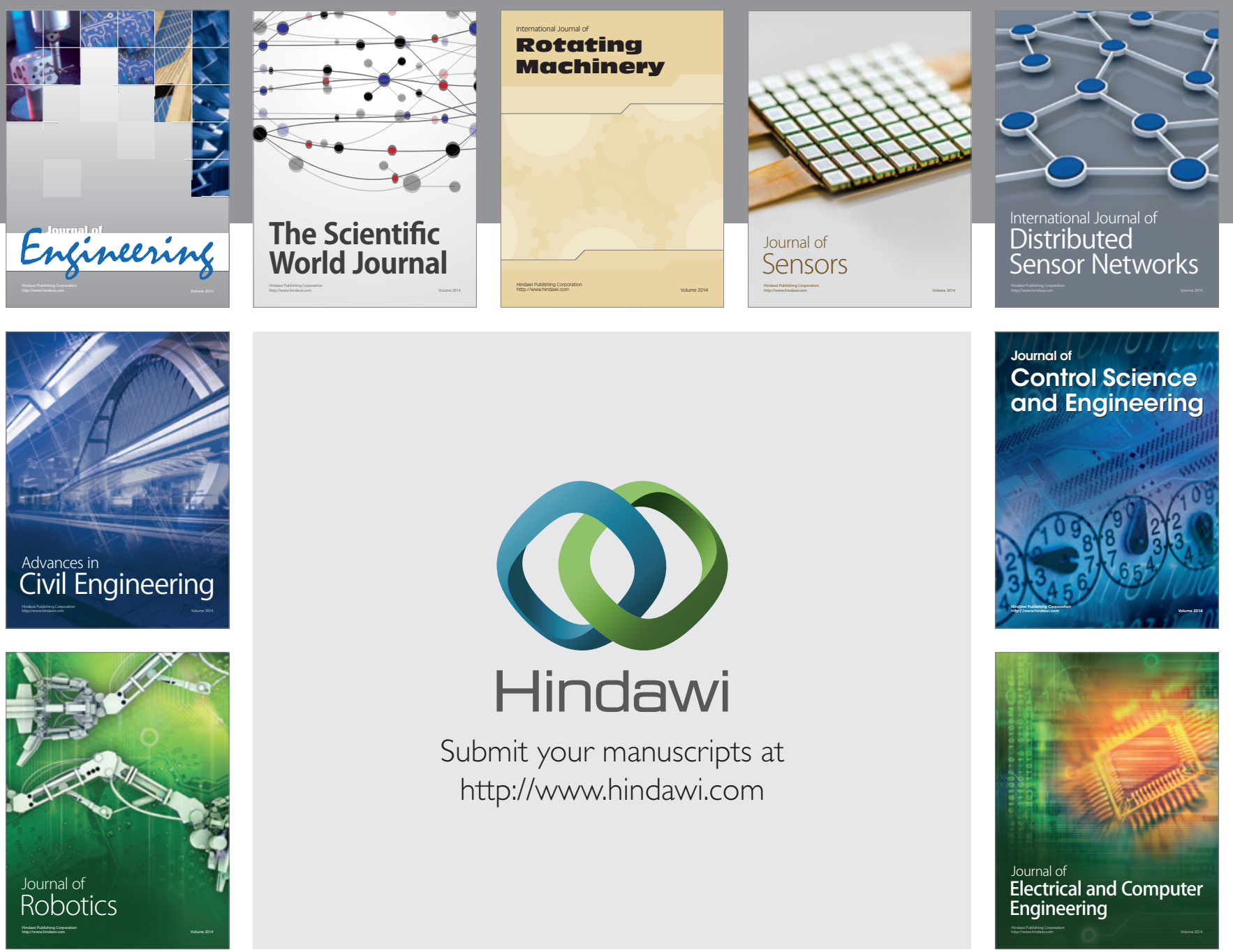

Submit your manuscripts at

http://www.hindawi.com
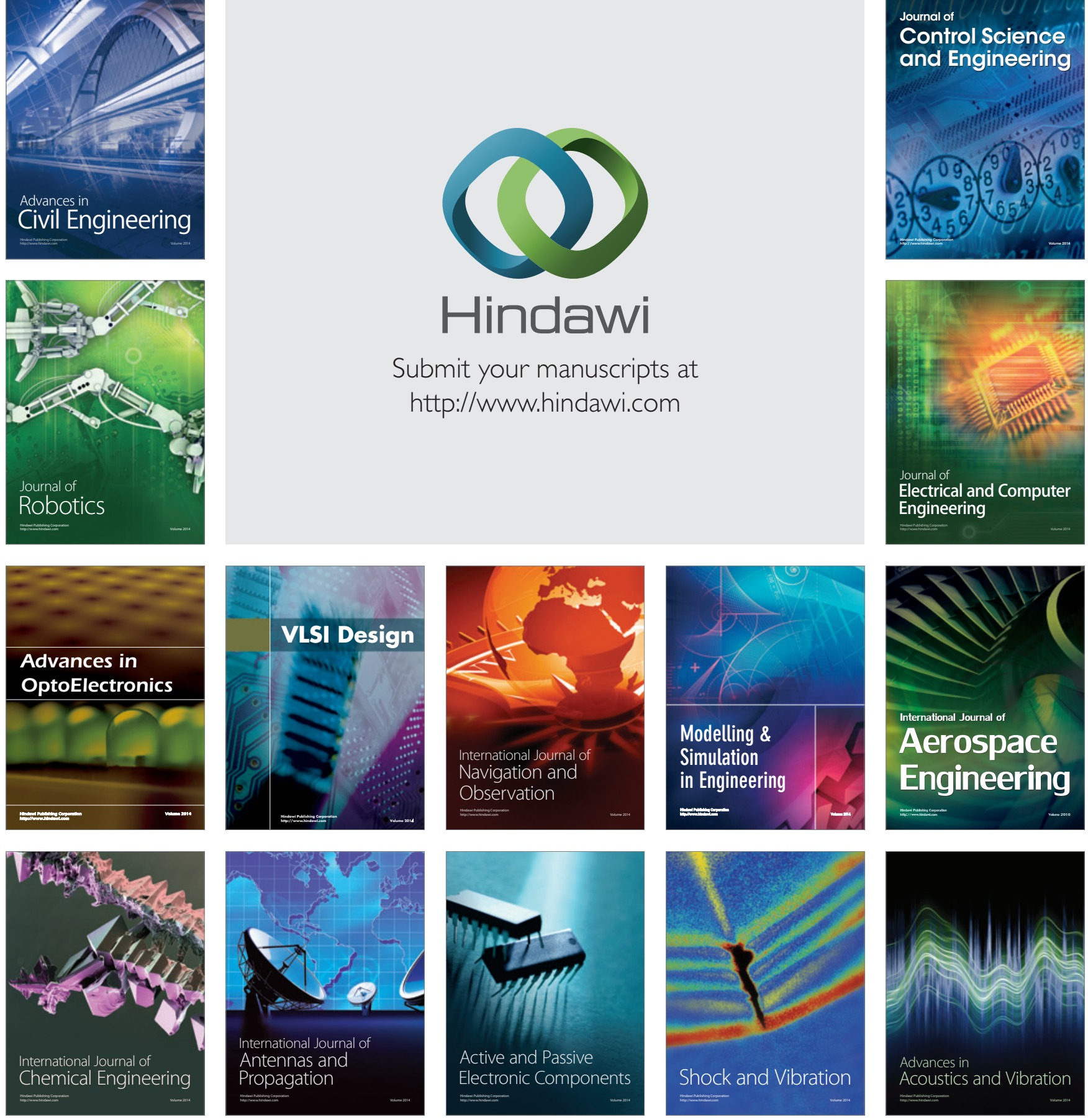